\title{
Simulate Heart Rate Variability in Different Physiological Conditions
}

\author{
DC Lin \\ Department of Mechanical, Aerospace \& Industrial Engineering, \\ Ryerson University, Toronto, Ontario, Canada
}

\begin{abstract}
Heart rate variability is assumed to result from a multiplicative or cascade mechanism based on its multifractal property. Numerical results on the perturbation of the cascade are given to compare HRV under parasympathetic (PNS) and sympathetic (SNS) autonomic blockades. It was found that qualitative change of the HRV multifractal, which was observed in PNS blockade, is due to the change from a multiplicative to an additive mechanism.
\end{abstract}

\section{Introduction}

The random fluctuation of the interbeat time (RRi) interval has been widely studied by its power spectrum, which is known to consist of a narrow-band harmonic component and a broad-band fractal component [1]. Recent studies suggest that the scaling structure of the fractal component is highly nonuniform and can in fact be put in the framework of multifractal [2,3]. Even for practical purposes, the fractal component of HRV can be of particular interest. It is known in healthy humans that the fractal signal power can measure up to $70 \%$ to $90 \%$ of the total RRi signal power [4]. Moreover, experimental data indicated strong correlation between the increase of mortality rate in certain heart disease population and the diminishing fractal characteristics [4]. However, the physiological origin and purpose of the fractal HRV remain largely unclear.

HRV is mainly contributed by the fluctuating autonomic nervous system dynamics (ANS). The blockade of the sympathetic (SNS) and parasympathetic (PNS) branches of ANS have shown some very interesting results $[5,6]$. In particular, the fractal component can go through a qualitative change from multi- to monofractal transition (MMFT) in PNS blockade but only to remain similar in SNS blockade. In the current investigation, we are interested in finding the leading factor of the cascade, which results in MMFT. In what follows, we will first propose the idea of cascade
HRV model based on the multifractal paradigm from complex systems (Section 2). We then apply perturbations to investigate the "instability factor" which can lead to MMFT in the cascade-generated multifractal (Section 3). Interestingly, the model favours multiplicative rather than additive mechanism, which is counterintuitive since the latter is related to the notion of feedback. Finally, the summary of results and conclusion are given in the last Section.

\section{Cascade heart rate variability}

Although the indication of multifractal fluctuation in HRV does not spell out the detail in physiology terms, it does suggest a multiplicative or cascade generating mechanism for the fractal component of HRV. The objective of this Section is to summarize the ideas of the cascade HRV.

Random cascade can be generally put in the framework of positive martingale theory [7]. In practical terms, it consists of three elements: $(\boldsymbol{a})$ the branching rule, (b) the probability law of the cascade component and $(\boldsymbol{c})$ the multiplicative data generation rule.

The cascade HRV assumes the random phenomenon is a result of the product of $\mathrm{J}$ cascade components:

$$
r_{J}(t)=\prod_{j=1}^{J} \omega_{j}(t)
$$

A gaussian $\omega_{j}(t)=1+\xi_{j}$ will be used through out this study where $\xi_{j}, j=1, \ldots, \mathrm{J}$ are independent gaussian variables (in $j$ ) with $\left\langle\xi_{j}>=0\right.$ and $\left\langle\xi_{i} \xi_{j}>=\delta_{i j} \sigma_{j}^{2}\left(\delta_{i j}\right.\right.$ is the Kronecker delta). It is necessary to assume a bounded $r_{J}(t)$ because of the refractory property of the heart muscle cells [8]. We impose a stronger condition in the model to further assure the boundedness in $J \rightarrow \infty$ limit. This can be achieved by letting $\sigma_{j}>\sigma_{j+l}$. Motivated by the deterministic bounded cascade [9], $\sigma_{j}=\sigma_{0} 2^{-\alpha(j-1)}$ were used where $\sigma_{0}$ and $\alpha$ are constants. The estimation of these parameters from the RRi data has been summarized 
in an article published in the same volume and in previous works [3].

In addition to the gaussian assumption and the decaying variance, $\omega_{j}(t)$ is further set to vary only on discrete times $\left\{t_{k}^{(j)}\right\}: \omega_{j}(t)=\omega_{j}\left(t_{k}^{(j)}\right)$ for $t_{k}^{(j)} \leq t<t_{k+1}{ }^{(j)}$. The time set $\left\{t_{k}^{(j)}\right\}$ is designed to provide the self-similarity structure in the data fluctuation and thus defines the branching rule for the cascade. In this work, dyadic time scales were used: $t_{k}^{(j)}=k N / 2^{j}, k=1, \ldots, 2^{j}$ where $N=2^{J}$ is the number of data points. Notice that the number of the elements of $\left\{t_{k}^{(j)}\right\}$ is an increasing function of $j$. Hence, the $\omega_{j}(t)$ for small $j$ can be considered as contributing to the large time scale fluctuation in HRV, or the "slow dynamics." Similarly, the $\omega_{j}(t)$ of large $j$ is considered as contributing to the small time scale fluctuation of HRV, or the "fast dynamics." It is tempting to relate such "slow" and "fast" dynamics to, respectively, the sympathetic and parasympathetic nervous system dynamics. Such a connection was recently tested and consistent results can indeed be established [10].

\section{Perturbed cascade HRV}

Regular cascade was able to generate very similar phenomenology observed in healthy daytime HRV [3]. To simulate HRV in different physiological conditions, the components $(\boldsymbol{a})$ and $(\boldsymbol{c})$ of the cascade are perturbed. The gaussian $\omega_{j}(t)$ will be used through out this study as other distributions do not seem to produce qualitatively different results. We then examine the scaling of the perturbed $r_{J}(t)$ in $\left\langle\left|\Delta r_{J}(\tau)\right|^{q}\right\rangle \sim \tau^{\zeta(q)}$ where $\Delta r_{J}(\tau)=r_{J}(t+\tau)$ $-r_{J}(t)$. For monofractal, $\zeta(q)=h q$ is a linear function and for multifractal, $\zeta(q)$ is nonlinear. In what follows, we will need to make reference to the interval length of the $j{ }^{\text {th }}$ generation of a dyadic cascade. For convenience, this is denoted by $\tau_{k, d}^{(j)}=2^{J-j}$.

The branching rule of the cascade is defined by three parameters $\left(C, \tau_{k}^{(j)}, N_{j}\right)$ : where $t_{k^{\prime}}^{(j+1)}=t_{k}^{(j)}, k^{\prime}=C k$, $\tau_{k}^{(j)}=t_{k+1}^{(j)}-t_{k}^{(j)}$ and $N_{j}$ denotes the number of intervals in the $j^{\text {th }}$ cascade generation. $\tau_{k}^{(j)}$ and $N_{j}$ are dependent to each other since $N=\sum_{k=1}^{N_{j}} \tau_{k}^{(j)}$. For example, $C=2$ and $\tau_{k}^{(j)}=\tau_{k, d}^{(j)}$ apply to the dyadic cascade, in which case, $t_{2 k}^{(j+1)}=t_{k}^{(j)}$ and $N_{j}=2^{j}$. The perturbation on the branching rule can thus be achieved by considering $C$ and $\tau_{k}^{(j)}$ as random variables. Fig. 1 shows the cascade configurations with the perturbed branching rule: a random $\tau_{k}^{(j)}$ of the dyadic cascade (Fig. 1a), a mixed dyadic and triadic cascade $(C=2$ and $C=3$ of equal probability) (Fig. 1b) and, more generally, a random $C$ constrained by $N_{j} \leq 3 \times 2^{j}$ (Fig. 1c). The averaged scaling exponent $\zeta(q)$ from 100 samples of $r_{J}(t)$ in respective cases show qualitatively similar shapes which indicate multifractal (Fig. 2). This implies the robustness in the cascade-generated multifractal under the branching rule perturbation.

It turns out a qualitative change of the scaling property is only possible by perturbing the data generation rule $(\boldsymbol{c})$. This is achieved by replacing (1) with

$$
r_{j}(t)=r_{j-l}(t)+\omega_{j}(t)
$$

for $t$ in randomly selected interval $\left[t_{k+1}^{(j)}, t_{k}^{(j)}\right]$. In the numerical experiment, a random draw $\mathbf{B}$ was first made uniformly in $[0,1]$ to determine the random intervals to which (2) is applied. The multiplicative rule (1) was kept if, for some $D$, the criterion $\mathbf{B}>D$ is satisfied, otherwise, (2) was used. We tested three cases: uniform perturbation (UP) with a constant $D=D_{0}$ (Fig. 3a), small time scale perturbation (SSP) with $D=D_{0} j$, where the criterion is less likely passed for large $j$ in the higher generation of the cascade (Fig. 3b), and large time scale perturbation (LSP) with $\mathrm{D}=D_{0}(J-j)$, where the criterion is less likely passed for small $j$ in the lower generation (Fig. 3c). For UP and SSP, the averaged $\zeta(q)$ is closed to a linear function, indicating MMFT after the perturbation (Fig. 4). The averaged $\zeta(q)$ under LSP is nonlinear in shape and is qualitatively similar to the unperturbed $\zeta(q)$ (Fig. 4). These results are consistent to the experimental findings of MMFT in PNS blockade and multifractal HRV in SNS blockade. At least in the framework of bounded cascade, the present results imply that MMFT is likely caused by the perturbation of the data generation rule from a multiplicative to an additive one.

\section{Conclusion}

In this study, the cascade HRV under autonomic blockade is studied. Numerical simulations were able to generate MMFT when multiplicative cascade is replaced by an additive mechanism uniformly over all time scales and selectively over the small time scales. By the analogy of "slow" and "fast dynamics" in the cascade, these results are consistent with the experimental observations of HRV in PNS and SNS blockades. It has long been recognized that biological systems should rely on some form of feedback mechanism to maintain its homeostasis. The present results raise an interesting question about the fractal component of HRV since feedback is in nature an additive procedure. This study showed that the cardiovascular regulation "favors" a multiplicative law in generating the broad-band characteristics of healthy 
HRV. The replacement by an additive rule, which can lead to MMFT, is in fact "problematic" in the sense of some known physiological effects in PNS blockade [6].
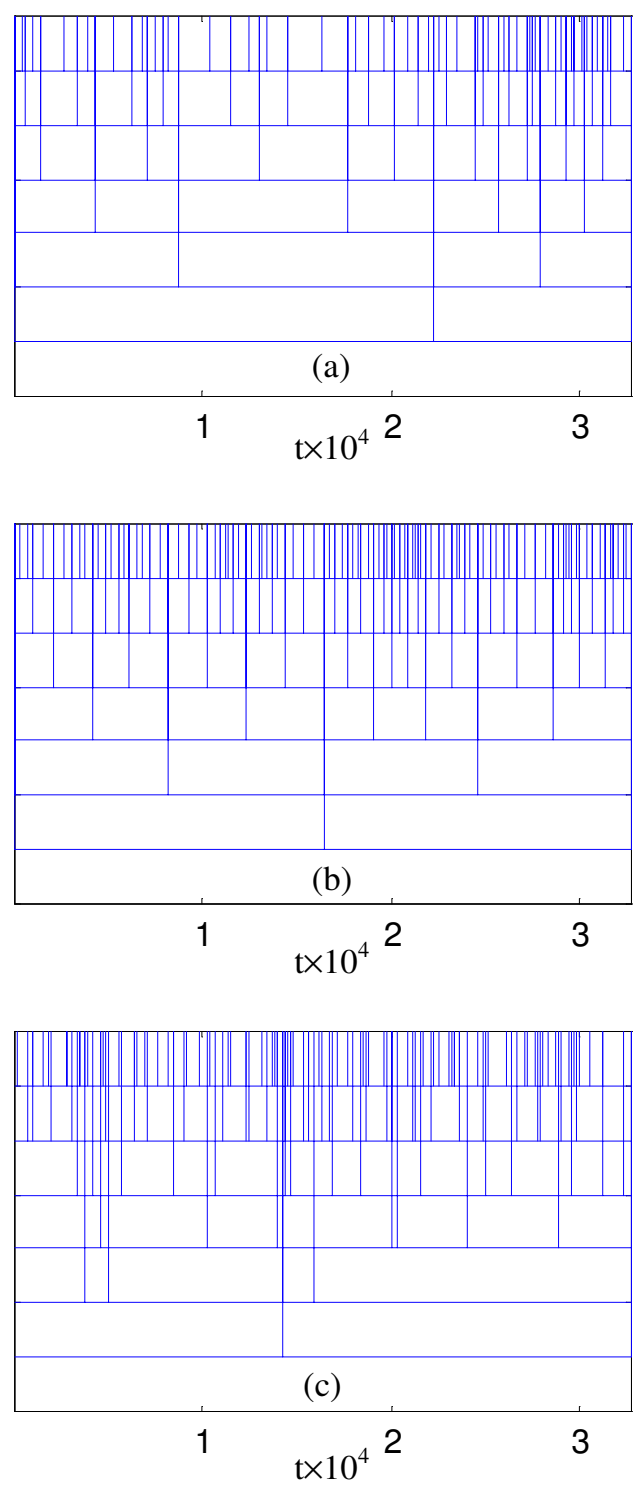

Figure 1 Cascade configurations from the branching rule perturbation. Only the first 6 generations are shown (The $0^{\text {th }}$ generation corresponds to initial condition): (a) $\tau_{k}^{(j)}=$ $(1+a \mathrm{U}) \tau_{k, d}^{(j)}$ and $\mathrm{U}$ is an uniformly distributed random variable in [0,1] and $a \in[0,1]$. (b) Mixed dyadic-triadic branching rule with $80 \%$ dyadic and $20 \%$ triadic branching. (c) Mixed branching rule where the number of interval at each generation is bounded by $N_{j} \leq 3 \times 2^{j}$ and $C$ is an integer random variable covering the full range bounded by $t_{k+1}^{(j)}-t_{k}^{(j)}$ for $j=1, \ldots, J$.

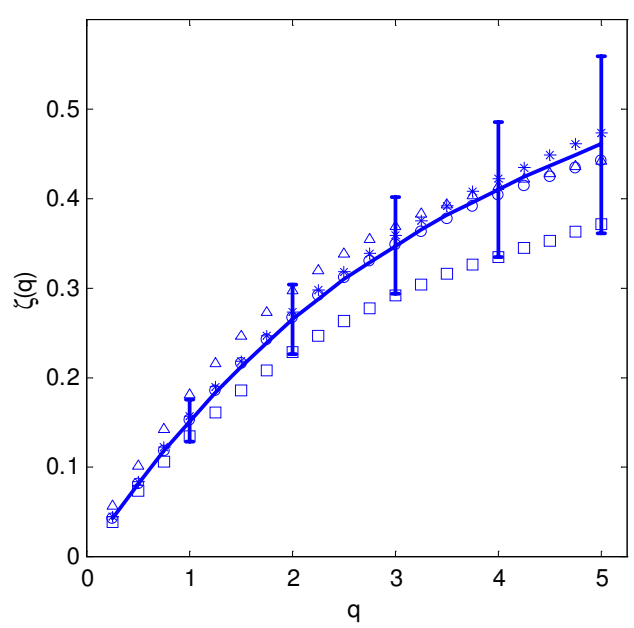

Figure 2 Scaling in the perturbed cascades shown in Fig.1: $\zeta(q)$ 's based on Fig. 1a for $a=0.4(0)$ and for $a=$ $0.8(\Delta)$, see also Fig. 1 caption; $\zeta(q)$ based on Fig. $1 \mathrm{~b}$ $(\square) ; \zeta(q)$ based on Fig. 1c (*). All $\zeta(q)$ are averaged from 100 samples of $r_{J}(t)$ using $\log _{2}\left(\sigma_{j}\right)=-1.6-0.126(j-1)$ and $J=15$. Solid line shows the averaged $\zeta(q)$ and one standard deviation for the unperturbed dyadic bounded cascade.

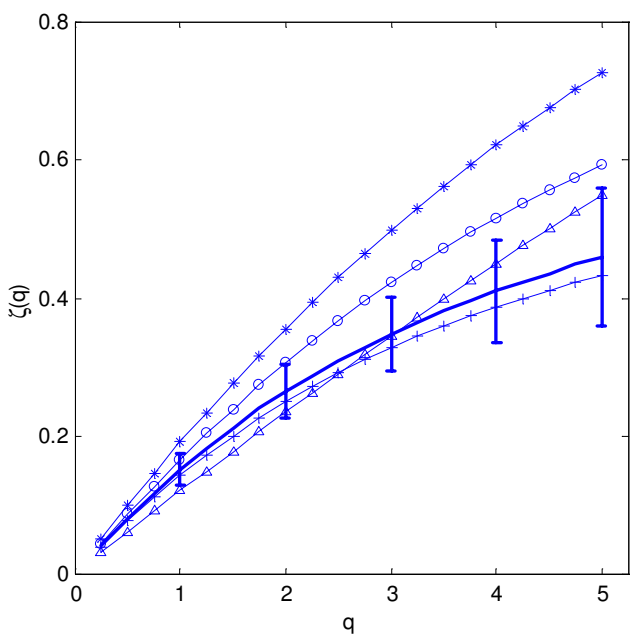

Figure 4 Scaling in dyadic cascades with perturbed data generation rule described in Fig. 3 (next page): $\zeta(q)$ for UP, $D_{0}=0.05(-+-)$ and $D_{0}=0.3(-*-) ; \zeta(q)$ for SSP, $D_{0}=0.03(-\Delta-)$; $\zeta(q)$ for LSP, $D_{0}=0.03(-0-)$. All $\zeta(q)$ 's are averaged from 100 samples of $r_{J}(t)$ based on $\log _{2}\left(\sigma_{j}\right)$ $=-1.6-0.126(j-1)$ and $J=15$. Solid line shows the averaged $\zeta(q) \pm$ one standard deviation for the unperturbed dyadic bounded cascade. 

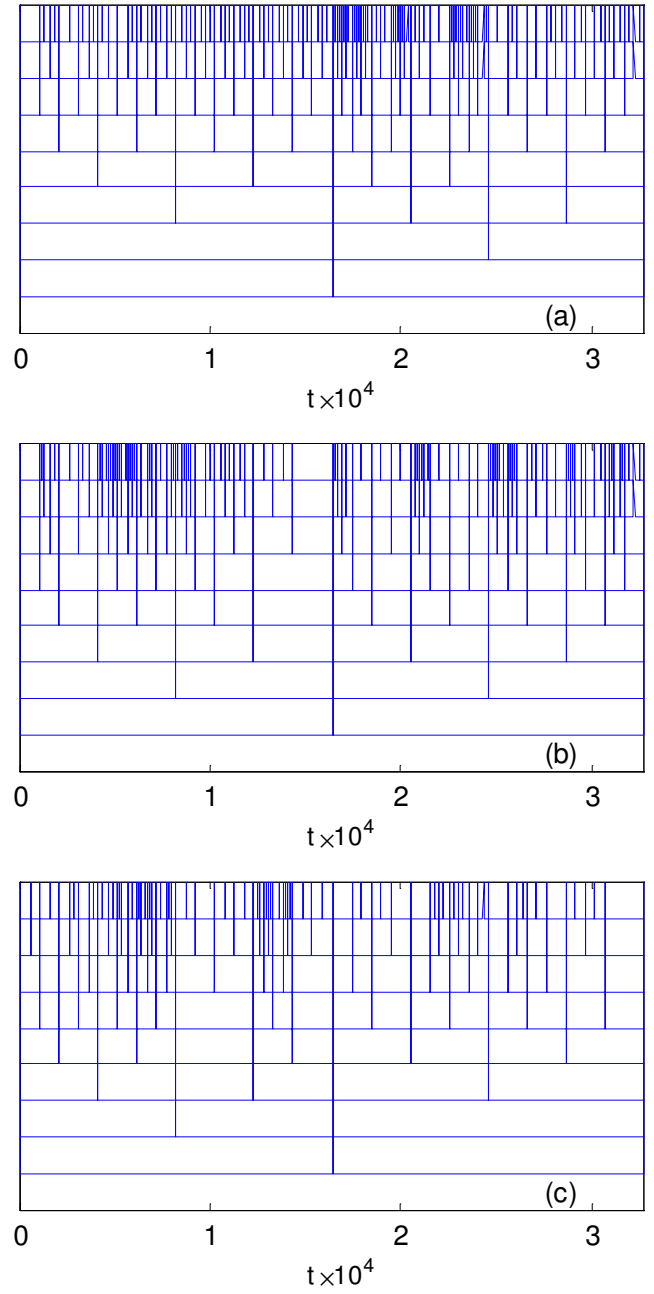

Figure 3 Representative cascade configurations for perturbed data generation rule. Only the first 8 generations are shown from bottom to top (the 0th generation is the initial condition). (a) UP, (b) SSP and (c) LSP; Only those intervals where the multiplicative rule (1) is applied are shown.

\section{Acknowledgements}

This research is supported by the Natural Science and Engineering Research Council of Canada.

\section{References}

[1] Yamamoto Y, Hughson RL. Extracting fractal component from time series. Physica D 1993;68: 250-264.

[2] Ivanov PCH, Amaral LAN, Goldberger AL, Havlin S, Rosenblum MG, Struzik, ZR, Stanley HE. Multifractality in human heartbeat dynamics. Lett. to Nature 1999;399:461465.

[3] Lin DC, Hughson RL. Modeling heart rate variability in healthy humans, a turbulence analogy. Phys. Rev. Lett., 86; 2001:1650-1653; Lin DC, Hughson RL. A phenomenology model for normal sinus rhythm in healthy humans. IEEE Tran. Biomed. Engng. 2002;49:97-114.

[4] Task Force of the ESC and NASPE. Standards and measurements of heart rate variability. Euro. Heart J. 1996;17:354-379.

[5] Amaral LAN, Ivanov PCH, Aoyagi N, Hidaka I, Tomono H, Goldberger AL, Stanley HE, Yamamoto Y. Behavioualindependent features of complex heartbeat dynamics. Phys. Rev. Lett. 2001;86:6026-6029.

[6] Yamamoto Y, Hughson RL. On the fractal nature of heart rate variability in humans: effects of data length and badrenergic blockade. Am. J. Physiol. Regul. Integr. Comp. Physiol. 1994;266: R40-R49; Y. Yamamoto, Y. Nakamura, H. Sato, M. Yamamoto, K. Kato, RL. Hughson. On the fractal nature of heart rate variability in humans: effects of vagal blockade. Am. J. Physiol. Regul. Integr. Comp. Physiol. 1995;269:R830-R837.

[7] Waymire EC, Williams SC. A general decomposition theory for random cascades. Bull. Am. Math. Soc. 1994; 31:216-222.

[8] Little RC and Little WC. Physiology of the Heart and Circulation. Year Book Med. Publ. Inc.; 1989.

[9] Marshak A, Davis A, Cahalan C, Wiscombe W. Bounded cascade models as nonstationary multifractals. Phys. Rev. E 1994;49:55-81.

[10] Lin DC. Model the fractal component in heart rate variability as a dyadic bounded cascade. Fractals 2002, to appear.

Address for correspondence.

D.C. Lin

Dept. of Mech, Aero. \& Ind. Engng.

Ryerson University

350 Victoria St.

Toronto, Ontario, Canada

derlin@ryerson.ca 